\title{
3D MORPHOLOGICAL ANALYSIS OF COMPOSITE MATERIALS WITH AGGREGATES OF SPHERICAL INCLUSIONS
}

\author{
ARnAud DELARUE AND DominiQue JEULIN
}

Centre de Morphologie Mathématique, Ecole des Mines de Paris, 35 rue Saint-Honoré, F-77305 Fontainebleau, France

e-mail: dominique.jeulin@ensmp.fr

(Accepted October 20, 2003)

\begin{abstract}
Composite materials containing aggregates of spherical inclusions are studied from 3D images obtained by $\mathrm{X}$-ray microtomography. Using two point statistics in different directions, and the empirical distribution of orientations of pairs of inclusions, interesting details concerning the anisotropy of the distribution of inclusions are obtained and are related to the method of construction for these materials. Some 3D morphological properties, available on the 3D images, give new information on the shape and the distribution of aggregates: tortuosity of shortest paths in the matrix, local volume fraction, geodesic distance function, local histograms of numbers of objects.
\end{abstract}

Keywords: covariance, geodesic distance, local number, local volume fraction, mathematical morphology, pole figures, second-order statistics, tortuosity.

\section{INTRODUCTION}

The aim of this work is to propose a morphological analysis of anisotropic structures containing aggregates of spherical inclusions. Such microstructures are very common in materials, and in the present case were studied in a joint research project (Delarue and Jeulin, 2000; Delarue 2001a). Composite materials were constructed from an Aluminium or a PMMA matrix incorporating Zircon or Zircon-Silica spherical inclusions. Materials were prepared in the GEMPPM (INSA de Lyon) and in the LTPCM (Grenoble). 3D images of these materials were obtained by X-ray microtomography in the Synchrotron of the ESRF (Grenoble) with a $2 \mu \mathrm{m}$ resolution per voxel.

We studied these structures by measurements on 3D images, such as the covariance and two point correlation functions of separate spheres in various directions, a tortuosity index obtained from 3D geodesic propagation, local volume fraction of inclusions, distance functions and angular distributions (to study the anisotropy of the neighborhood of inclusions). Finally, local histograms of the number of sphere centers in a given domain are estimated and interpreted.

\section{DESCRIPTION OF MATERIALS}

The studied composite materials are made with a PMMA or an Aluminum matrix and Zircon or ZirconSilica spherical inclusions with almost a single size (with a $50 \pm 10$ microns radius). Two processes were used (Fig. 1). In the first process, a powder of PMMA and Zircon-Silica is mixed in a turbula. It is heated to $450^{\circ} \mathrm{C}$ under a $20 \mathrm{MPa}$ pressure for 1 hour. Some of these composite samples (Fred materials) are then deformed by a compression with a 3:1 factor along the $X$ axis, with a free extension along the $Y$ axis. No extension is allowed along the $Z$ axis.

The second process is radically different. Aluminum powder (the matrix) and Zircon (inclusions) are mixed in a liquid environment. Aggregates of inclusions are formed by this method. This material is compressed along the $Z$ axis $(P=$ $\left.70 \mathrm{Mpa}, 450^{\circ} \mathrm{C}, 20 \mathrm{~min}\right)$. Some of them are extruded through a hole having a section with proportions $16: 1$ along the axis $Z$.
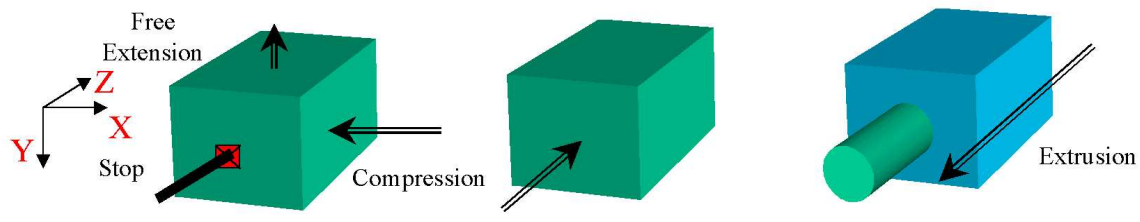

Fig. 1. Methods of preparation of materials. 
During the process, inclusions remain spherical, since they are much harder than the matrix. They may come in contact with each other, but do not overlap (however, as a consequence of the limited spatial resolution of images, some apparent overlaps can appear). Depending on the process, some degree of anisotropy in the arrangement of inclusions, as well as the formation of aggregates, may be expected. Morphological measurements were made to describe these points. In what follows, we are using estimations of some morphological functionals, which are defined for stationary random sets.

In this paper, we study six materials (Table 1). 3D images obtained by microtomography have sizes

around $300^{3}$ voxels, with a resolution of 4 microns per voxel and only two colors (images were binarised by a standard thresholding), from which are estimated the morphological properties.

Table 1. Studied materials.

\begin{tabular}{|c|c|c|}
\hline Name & Composition & Transformation \\
\hline Fred1 & PMMA + 40\% Zircon-Silica & Homogeneous \\
\hline Fred2 & PMMA + 40\% Zircon-Silica & Deformed \\
\hline Jmc2c & Aluminum + 20\% Zircon & Compressed \\
\hline Jmc2e & Aluminum + 20\% Zircon & Extruded \\
\hline Jmc3c & Aluminum + 35\% Zircon & Compressed \\
\hline Jmc3e & Aluminum + 35\% Zircon & Extruded \\
\hline
\end{tabular}

Table 2. Visualization of studied materials

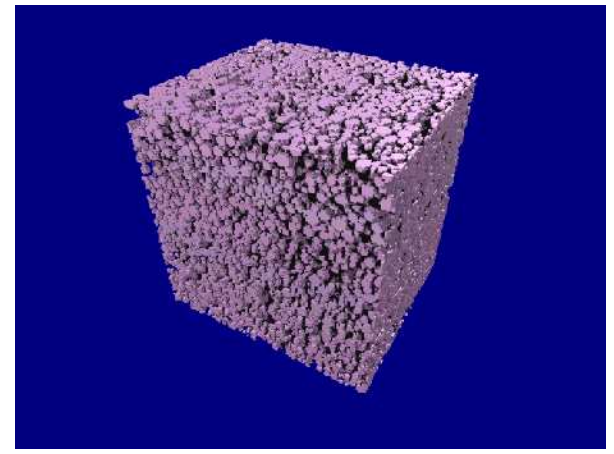

Material Fred1

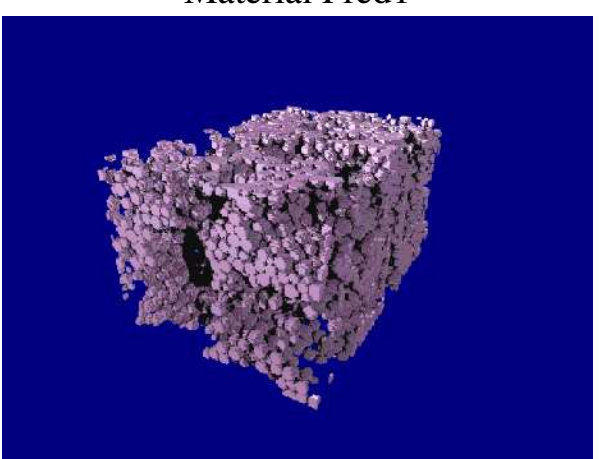

Material Jmc2c

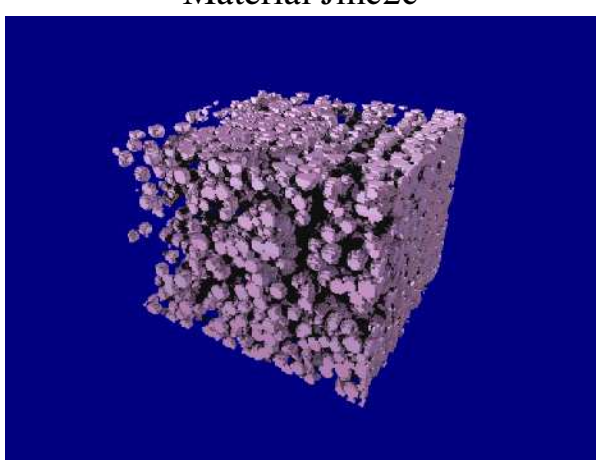

Material Jmc3c

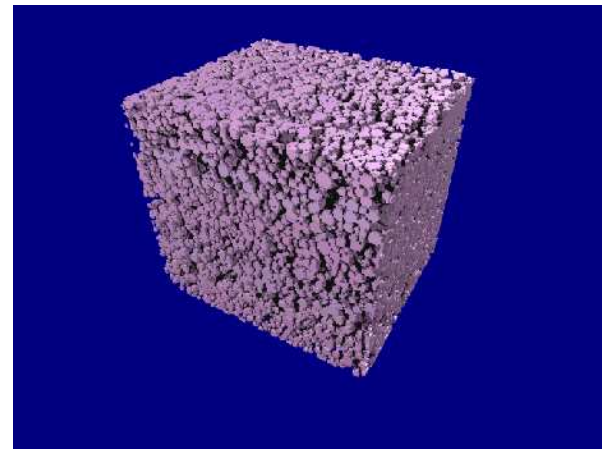

Material Fred2

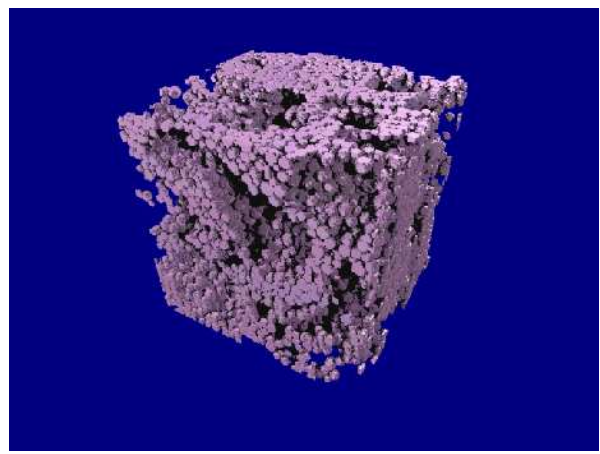

Material Jmc2e

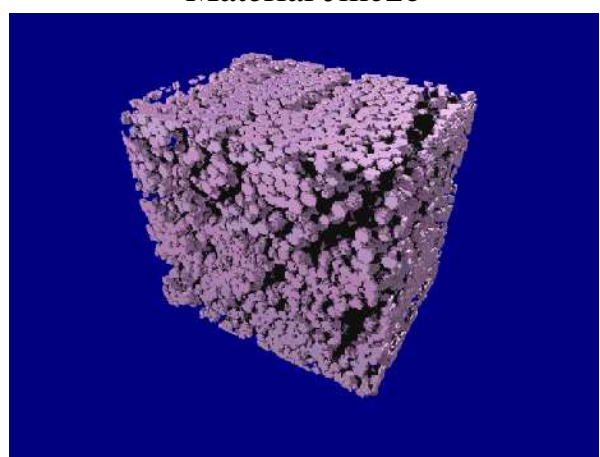

Material Jmc3e 


\section{GLOBAL ANALYSIS}

In this part, the morphology of inclusions and of aggregates is studied by measurements like the covariance, two point probability functions of separate spheres, distance function of inclusions in the matrix, and by the tortuosity of the matrix. They are measured on the whole $3 \mathrm{D}$ image.

\section{COVARIANCE}

The covariance $C(h)$ (Matheron, 1965, 1975; Serra, 1982) is the probability for two points, separated by the vector $h$ (with a modulus expressed in microns in the present case), to belong to the same stationary random set $A$. We have:

$$
C(h)=P\{x \in A, x+h \in A\}=P\left\{x \in A \cap A_{-h}\right\},
$$

which is estimated from the measurement of the volume fraction (deduced from the number of voxels in one set) of the intersection of the set $A$ and the translate of $A$ by the vector $h$. This measurement is sensitive to the heterogeneity of the spatial distribution (like the presence of aggregates) and the anisotropy, provided the sample is representative of the investigated microstructure. When $h=0$, we have $C(h)=p$, the volume fraction of the set $A$ (here inclusions). If the events $\{x \in A\}$ and $\{x+h \in A\}$ become independent as the magnitude of $h$ becomes large, then $C(h)$ converges to $p^{2}$ as $h$ increases. The way $C(h)$ changes from $p$ to $p^{2}$ is indicative of the structure of the material. Fig. 2 shows the sample covariance for the materials prepared according to the first process (Fred1 and Fred2). These materials should be isotropic, but material Fred 2 shows a slight anisotropy, which is indicated by a different empirical covariance in the direction of the $Y$ axis. From Fig. 2, it seems that the sample correlations disappear when the separation reaches the average diameter of inclusions. Hence, we can conclude that there is no dependence of arrangement of inclusions to a scale larger than their diameter; that is, the spatial arrangement of the inclusions involves a single scale, namely that of the individual inclusions.
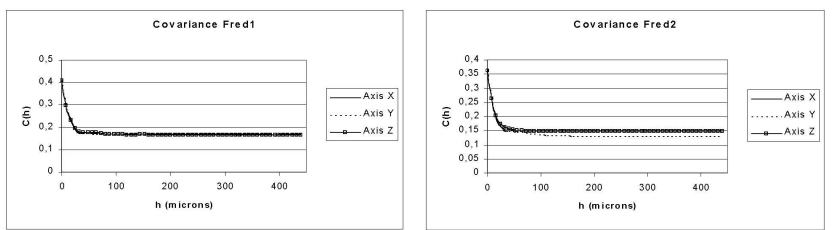

Fig. 2. Empirical covariance for homogeneous materials

Compressed aggregates materials have a very different behaviour. Fig. 3 shows the covariance of the materials $\mathrm{Jmc} 2 \mathrm{c}$ (20\% of inclusions) and $\mathrm{Jmc} 3 \mathrm{c}$
(35\% of inclusions). We can discern a second scale of order, introduced by aggregates. It is interesting to notice that they are elongated orthogonally to the $Z$ axis. This anisotropy is induced by the compression of the composite along this axis.
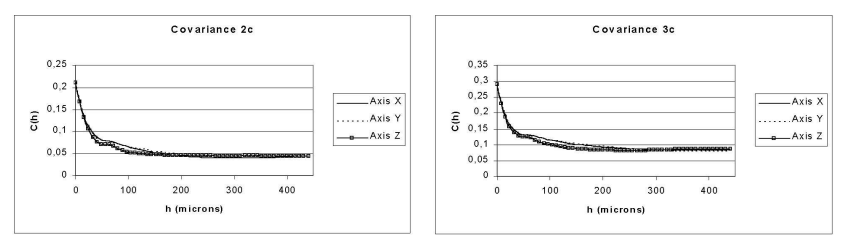

Fig. 3. Empirical covariance for aggregate materials

The extruded materials show a completely different covariance (Fig. 4). Aggregates disappear, a slight gap appears on the $Z$ axis and a long distance order appears on the structure. The gap is the sign of a repulsion between neighbor inclusions. We can expect that the extrusion destroyed aggregates and aligned inclusions along the extrusion axis $Z$. The alignment can involve a long range order.

Finally, all experimental covariances reach their sill for a distance, or correlation length, much smaller than the size of the studied field. As a result, we can accept the assumption that the studied 3D images are realizations of stationary random sets. We could also use the covariance to estimate the variance of estimation of the volume fraction of particles, as developed in geostatistics (Matheron, 1965).

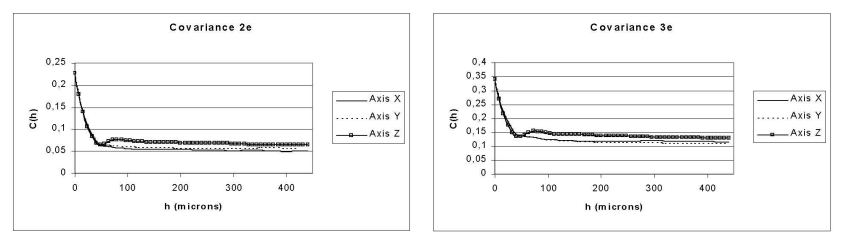

Fig. 4. Empirical covariance for extruded materials

\section{TWO POINT PROBABILITY FUNCTION OF DISTINCT SPHERES}

For this kind of composite material, where the structure is made of inclusions and aggregates of inclusions, it is interesting to obtain two point probability functions $P_{2}(h)$ involving distinct inclusions $A_{1}$ and $A_{2}$ :

$$
P_{2}(h)=P\left\{x \in A_{1} ; x+h \in A_{2}\right\} .
$$

This probability does not depend on $x$ for a stationary random distribution of inclusions, which is assumed below. It can be estimated on images by an algorithm where inclusions are labelised (Jeulin, 2001). But there is a simpler method applicable for structures with convex elementary grains. In that case, 
the probability $P(h)=P\{h \subset A\}$ for the full segment $h$ to be included in inclusions is the same as the probability for points $x$ and $x+h$ to be in the same inclusion. Therefore, the probability $P_{2}(h)$ can be directly estimated as the expression $C(h)-P(h)$.

For all populations of non overlapping objects, $P_{2}(0)=0$ (the probability for one point to be in two separate inclusions is zero). When $h$ increases, $P_{2}(h)$ moves to $p^{2}$, exactly like the covariance. The way $P_{2}(h)$ changes to $p^{2}$ is, like for the covariance, typical of the arrangement of inclusions in space (while the function $P(h)$ is only sensitive to the size distribution of inclusions).

We present now some experimental results. For homogeneous materials, like Fred1 (Fig. 5 left), the empirical $P_{2}(h)$ increases monotonously from 0 to $p^{2}$. We can conclude surely that there is no large scale dependence of inclusions in that case. $P_{2}(h)$ measurement for the deformed material Fred2 (Fig. 5 right) is different. A slight attraction is detected between grains, even for a long distance for the axis $X$ and $Z$. For $h$ parallel to the $Y$ axis, the empirical two-point probability has the expected $p^{2}$ asymptote. This phenomenon can be explained by the anisotropy of the deformation. Inclusions compressed along the $X$ axis cannot expand along $Z$ (for which no extension is permitted), but can expand along the $Y$ axis. In this case, inclusions support attraction and local alignment because of the high density (40\%), but the structure remains disordered along the free axis $Y$.
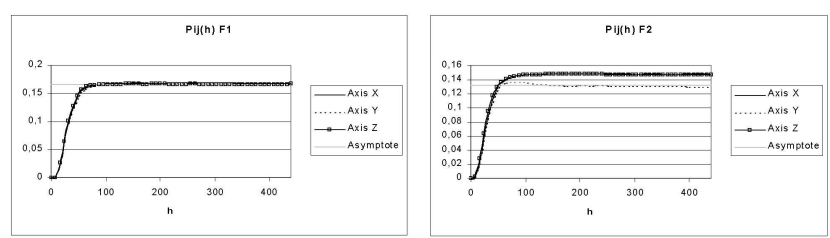

Fig. 5. Empirical two point probability function $P_{2}(h)$ of homogeneous materials

Compressed structures are rather different (Fig. 6). The estimated probability to find an inclusion close to a second one is quite larger than $p^{2}$. It is an attraction effect, typical of aggregates and here, it is possible to discern more clearly than with the covariance the size of aggregates and the anisotropy. Aggregates have a 150 microns length along the $Z$ axis and a 200 microns $(\mathrm{Jmc} 2 \mathrm{c})$ or 300 microns $(\mathrm{Jmc} 3 \mathrm{c})$ length along the $X$ and $Y$ axis. They are flattened as a result of the compression.
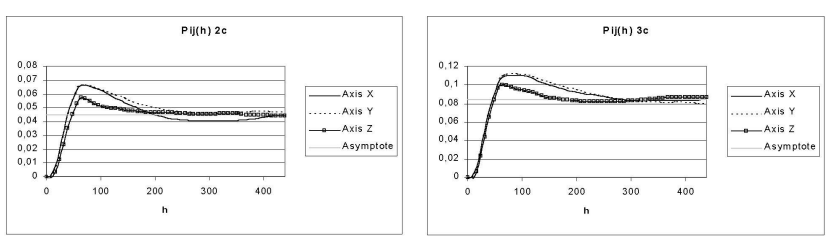

Fig. 6. Empirical two point probability function $P_{2}(h)$ of aggregated materials

Extruded materials present another behaviour (Fig. 7). The estimate of $P_{2}(h)$ monotonously grows to an asymptote (close to $p^{2}$ for the axis $X$ and $Y$ ), higher than $p^{2}$ for the axis $Z$. This is caused by the alignment resulting from the extrusion, destroying aggregates and aligning inclusion along the $Z$ axis with an attraction effect and a slight repulsion for the $X$ and $Y$ axis.
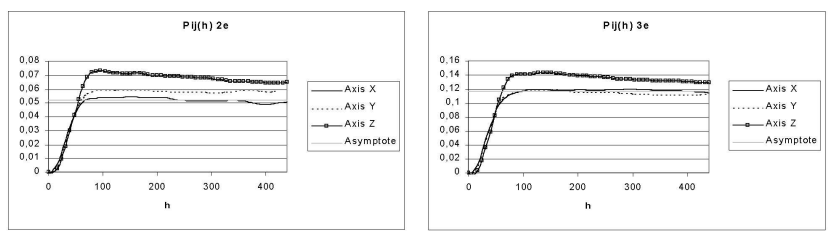

Fig. 7. Empirical two point probability function $P_{2}(h)$ of extruded materials

\section{TORTUOSITY}

The tortuosity in every point $x$ of the matrix can be defined from the distance of shortest paths through $x$ in the matrix (inclusions have to be avoided) connecting two opposite faces of the 3D block, normalized by the distance between the faces (it is always larger than or equal to1) (Decker et al., 1998). The Fig. 8 is a 2D representation of the tortuosity of one point:

$$
\operatorname{Tortuosity}(x)=\frac{d_{1}(x)+d_{2}(x)}{D} .
$$

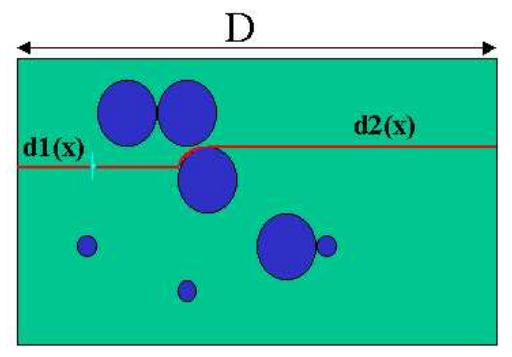

Fig. 8. Shortest path containing a point $x$ in the matrix, connecting two opposite edges of the image

Measurements presented here are obtained for every voxel of the grid in 3 dimensions in the three main directions $(X, Y, Z)$. For Fred materials (Fig. 9) the empirical distributions of tortuosity show a nearly Gaussian shape centered on 1.05. This is the characteristic value for a homogeneous distribution 
of spheres in space with a $40 \%$ volume fraction. The compressed and extruded materials with $20 \%$ of inclusions are too low density material for this study, showing a very high proportion of points with a tortuosity equal to 1. , as a result of a majority of straight paths through the matrix. The other compressed and extruded materials, with a $35 \%$ volume fraction of inclusions are more interesting to study. The compressed one (Jmc3c) shows a tortuosity lower than 1.03 for the $X$ and $Y$ axis and slightly higher than 1.03 for the $Z$ axis (Fig. 10). This can be explained by the shape of aggregates, elongated orthogonally to the $Z$ axis. The tortuosity is larger on the $Z$ axis because it is more difficult to go round flat domains in the $Z$ direction than along them on the $X$ or $Y$ direction.
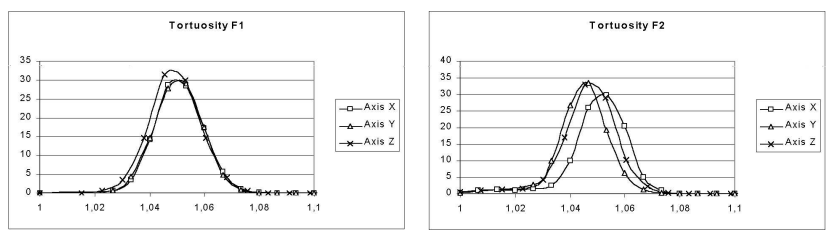

Fig. 9. Histogram of tortuosity for heterogenous materials

This difference is more spectacular with extruded materials. The tortuosity along the $X$ and $Y$ axis is slightly higher. This is the result of the aggregates destruction by extrusion. Homogeneous structures have a higher tortuosity than compressed structures. The extrusion involves also an alignment along the $Z$ axis. Its effect on the tortuosity measurement appears clearly. A large part of the matrix points own a straight path connecting the two faces orthogonal to the $Z$ direction (tortuosity equal to 1 ).
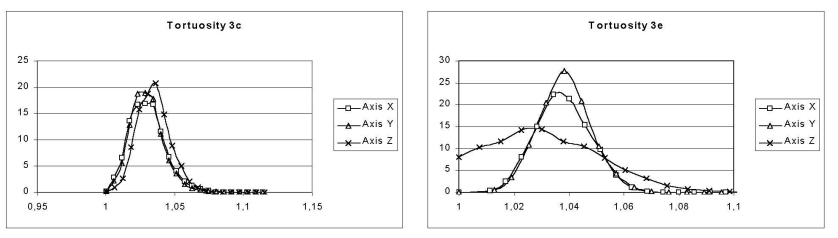

Fig. 10. Histogram of tortuosity for aggregated and extruded materials

\section{CONCLUSION}

With only two types of measurements, the two point probability function of separate inclusions (showing here more details than the covariance), and the tortuosity, we clearly make the difference between homogeneous structures, compressed structures and structures with alignments in one direction. For the six studied materials, these measurements help us to understand the consequence of the process, mainly the deformation of aggregates under compression and the aggregate destruction followed by an alignment of inclusions by the extrusion.

\section{LOCAL ANALYSIS}

Local measurements are presented in this part. A method based on Voronoï zones of influence is used to obtain local volume fractions. A comparison between 2D and 3D measurements is presented. The second paragraph presents the geodesic distance function of inclusions. The last paragraph gives an angular empirical distribution function of pairs of inclusions, which helps to understand local rearrangements occurring during the manufacturing process.

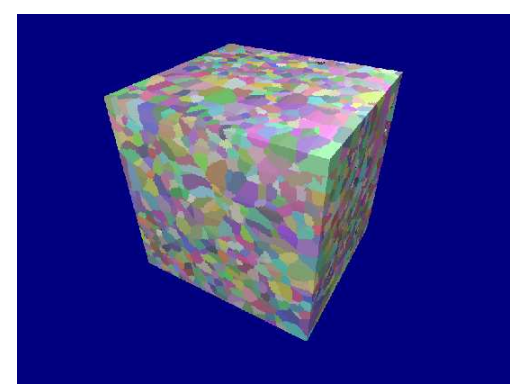

Fig. 11. Voronoï zones of influence of a composite material with hard spheres

\section{LOCAL VOLUME FRACTION}

The local volume fraction is defined by ratio between volumes of each inclusion and of its zone of influence in 3D. The most difficult part of the work is to obtain the zone of influence of every inclusion. These zones are obtained in three steps. The first step completely separates inclusions, which may show slight overlaps due to the low spatial resolution of images. This is obtained by a small $3 \mathrm{D}$ cubic erosion. Its size is selected in such a way that the smallest inclusions are prevented to disappear. The right size of erosion is obtained when we get the experimental maximum of the connectivity number, since it increases when separating overlapping objects, and it decreases when suppressing objects. Separate inclusions are labelised in a second step. Finally a label propagation is applied on inclusions to obtain their Voronoï zones of influence (Fig. 11). Each obtained polyhedron is a zone of influence of a single inclusion. Then, the empirical distribution function of the volume of each inclusion gives us a size distribution (Fig. 12). In addition, the histogram of the local volume fraction of inclusions is compared to the analogously defined local area fraction in zones of influence on 2D sections. Notice that the Voronoi zones of influence which refer to the $2 \mathrm{D}$ section profiles do not coincide with the intersections of the 3D zones of influence. 


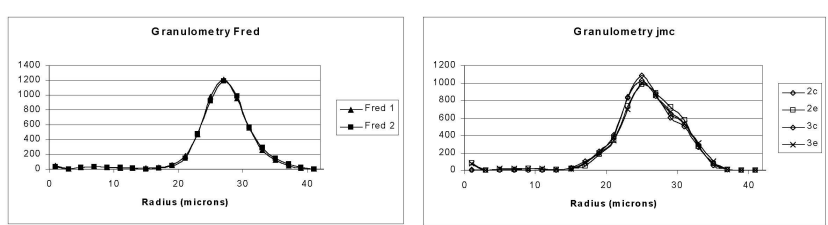

Fig. 12. Granulometry of Jmc and Fred materials

For homogeneous materials (Fig. 13), we get a unimodal histogram centered on the $40 \%$ volume fraction. For compressed materials (Fig. 14), aggregates involve a change of the histogram, which becomes skewed or bimodal. Grains included inside aggregates have a very high volume fraction, as opposed to inclusions present on the surface of the aggregates, showing a low volume fraction. Extruded structures with inclusion alignments show a skewed histogram (Fig. 15). It is easy to understand that the destruction of aggregates by the extrusion limits the range volume of volume fractions. It is interesting to notice than $3 \mathrm{D}$ measurements can be quite different from 2D measurements, mainly for these last materials. The difference between 2D and $3 \mathrm{D}$, more pronounced on anisotropic structures, mainly appears on the tails of the histograms, longer in $3 \mathrm{D}$ than in $2 \mathrm{D}$.
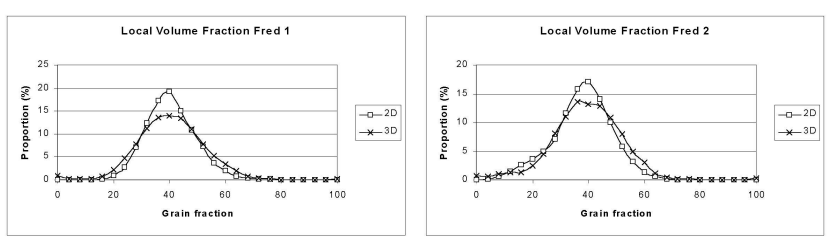

Fig. 13. Histograms of local volume fractions of homogeneous materials

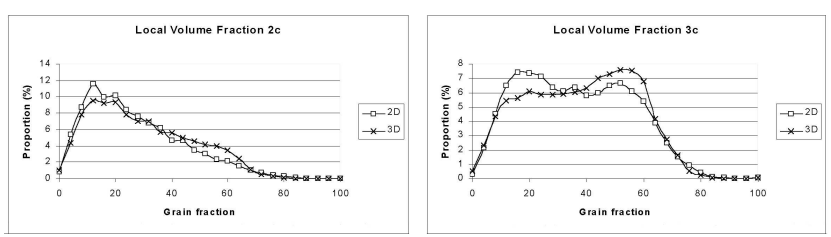

Fig. 14. Histograms of local volume fractions of aggregated materials

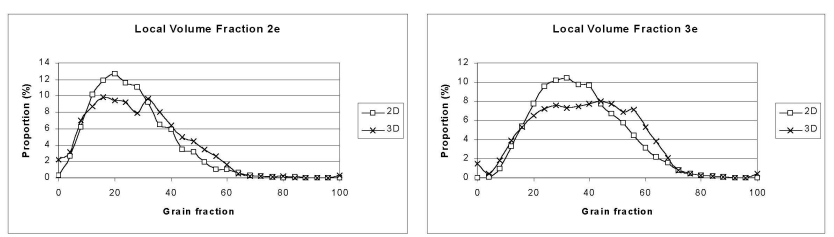

Fig. 15. Histograms of local volume fractions of extruded materials

\section{GEODESIC DISTANCE FUNCTION}

This measurement is obtained by 3D geodesic dilatations of inclusions in the matrix. We use the empirical distribution function of distances, which is sensitive to the distribution of sizes of the zones of influence. For homogenous structures, a one scale histogram appears on Fig. 16, inclusions being separated by distances with a low dispersion. For compressed and extruded materials (Fig. 17), the histogram of distances covers a wider range of values. For aggregated (compressed) materials (Fig. 17 left), two modes appear (the second one being rather weak), as a result of a structure presenting arrangements on two different scales, which confirms results of second order statistics. Extruded materials (Fig. 17 right) show a slightly more regular histogram, showing an intermediate structure situation between compressed and homogenous materials.

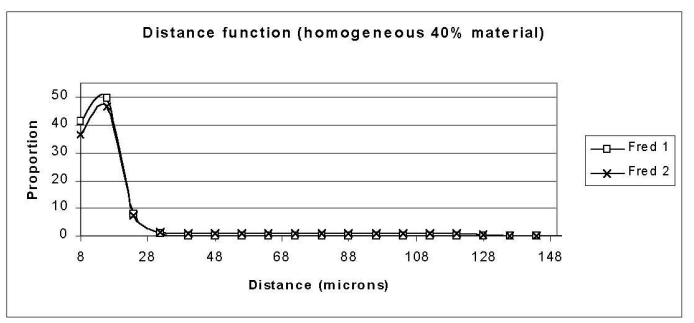

Fig. 16. Histogram of the distance function of a homogeneous material

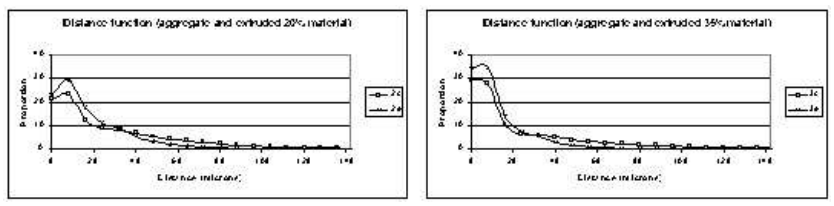

Fig. 17. Histograms of the distance function of aggregate and extruded materials

\section{ORIENTATION OF PAIRS OF INCLUSIONS}

Another type of measurement, which reflects the anisotropy on a very small scale, is obtained by the detection of the distance and of the angle made by each pair of centers of inclusions with a fixed direction. For every inclusion, the coordinates of its barycenter and its radius (as a function of its volume) are calculated. For each pair of centers of inclusions in contact (detected by comparing the distance between their centers to the sum of their radii), spherical angles $\phi$ and $\theta$ are measured (Fig. 18). A pole figure is obtained from the projection of each vector representing every pair of inclusions on a given plane. To get more details, the empirical distribution function of the angle $\phi$ can be plotted. 


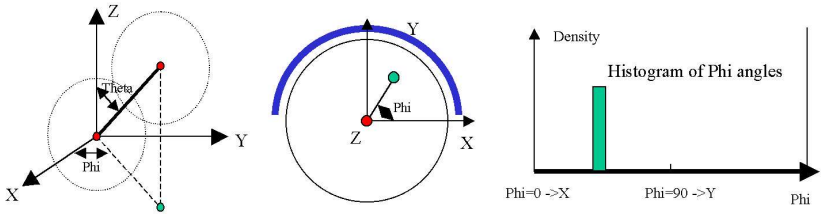

Fig. 18. Angle convention and pole figure of the orientation of pairs of grains

For the compressed materials with $35 \%$ of inclusions ( $\mathrm{Jmc} 3 \mathrm{c}$ et $\mathrm{Jmc} 3 \mathrm{e}$ ), this measurement is very interesting. The Figs. 19, 20 and 21 show that the structure does not present a high local anisotropy. There is a slightly higher population of pairs of inclusions oriented along the $Z$ axis, as a result of the compression.
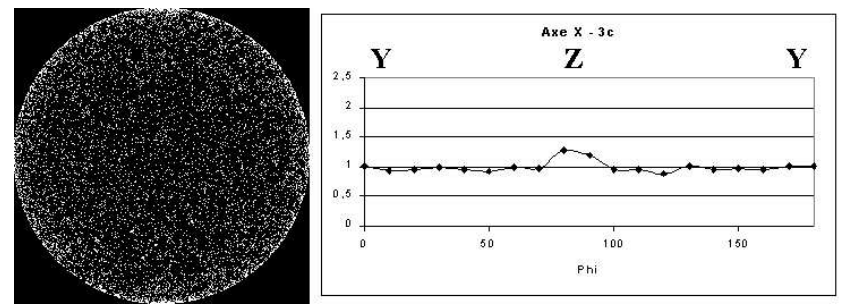

Fig. 19. Pole figure and projection, orthogonally to the $X$ axis (compressed 35\% material Jmc3c)
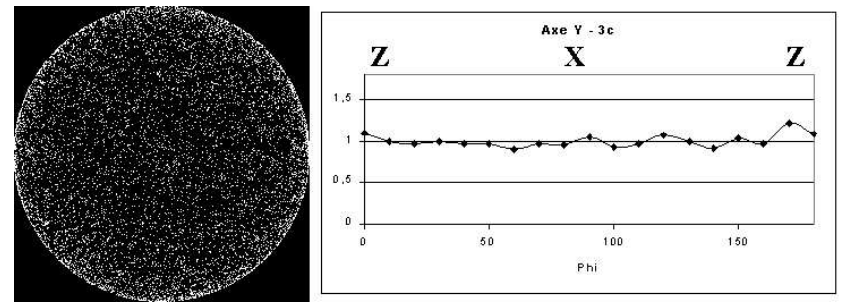

Fig. 20. Pole figure and projection, orthogonally to the $Y$ axis (compressed 35\% material $\mathrm{Jmc} 3 \mathrm{c}$ )
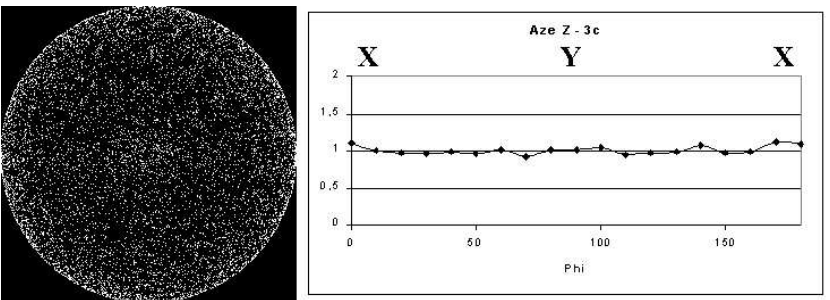

Fig. 21. Pole figure and projection, orthogonally to the $Z$ axis (compressed $35 \%$ material Jmc 3c)

Pole Figs. 22, 23 and 24 show that the extrusion enhances local orientation effects, due to the alignment of inclusions along the $Z$ axis: during the compression, inclusions turned around the axis $X$ towards the $Y$ axis. From other figures given in (Delarue and Jeulin, 2000), the compressed material with a $20 \%$ content of inclusions (Jmc2c) has almost the same empirical distributions as the $35 \%$ material. It shows a slight peak along the $Z$ axis, due to the compression, while for the same $Z$ axis there is a deficit of pairs of inclusions for extruded material Jmc2e. This slight gap can be explained by the inclusion removal when they were aligned during the extrusion.
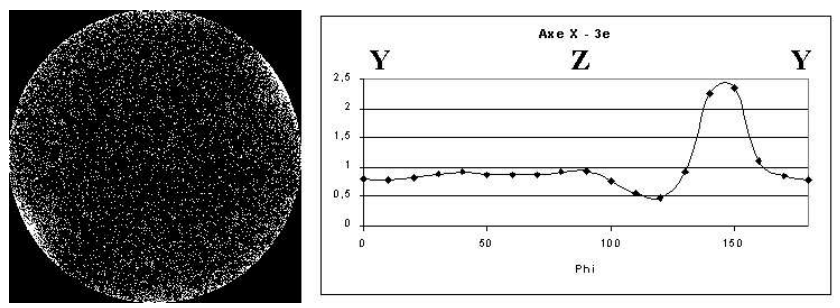

Fig. 22. Pole figure and projection, orthogonally to the $X$ axis (extruded $35 \%$ material $J m c 3 e$ )
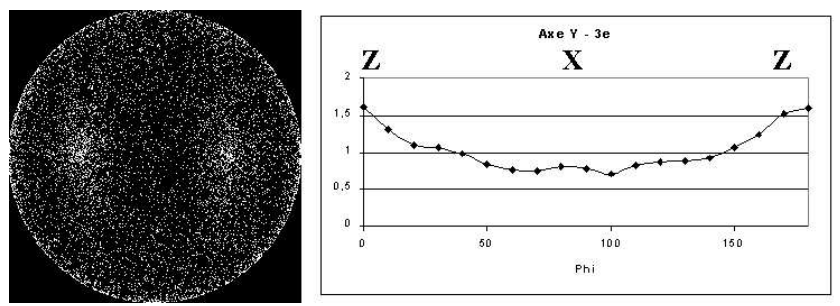

Fig. 23. Pole figure and projection, orthogonally to the $Y$ axis (extruded $35 \%$ material $\mathrm{Jmc} 3 e$ )
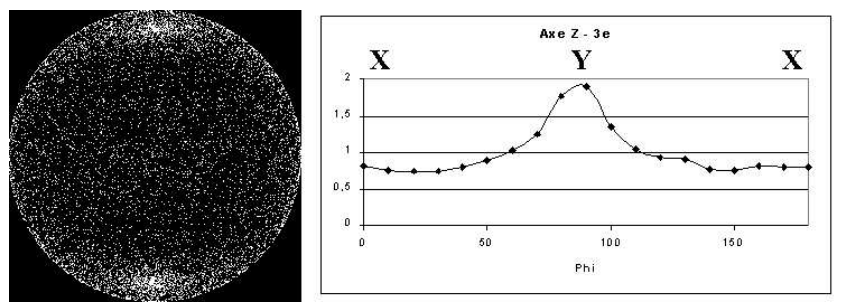

Fig. 24. Pole figure and projection, orthogonally to the $Z$ axis (extruded $35 \%$ material $\mathrm{Jmc} 3 e$ )

For the homogeneous Fred1 material, we discern that the process involves a slight compression along the $Z$ axis. The deformed material Fred2 shows the same effect along the $Z$ axis, but it appears that the compression along the $X$ axis forced inclusions to turn around themselves towards the $Y$ axis, which is the only free direction.

\section{CONCLUSION}

This part presented the results of local measurements. Local volume fractions and geodesic distance functions have confirmed the results of the global measurements presented before. The empirical distributions of orientations of pairs of inclusions bring more details about the manufacture process like collective movement of inclusions during the compression, the extrusion or the deformation under the influence of the density. 


\section{LOCAL HISTOGRAMS OF THE NUMBER OF SPHERE CENTERS}

In this part, we present a 3D analysis of granular arrangements based on the the histogram of the number of spheres included in a randomly located test set. To account for the anisotropy of the structure, this set is a parallelepiped with different orientations. Different sizes of parallelepiped are used to obtain information at various scales.
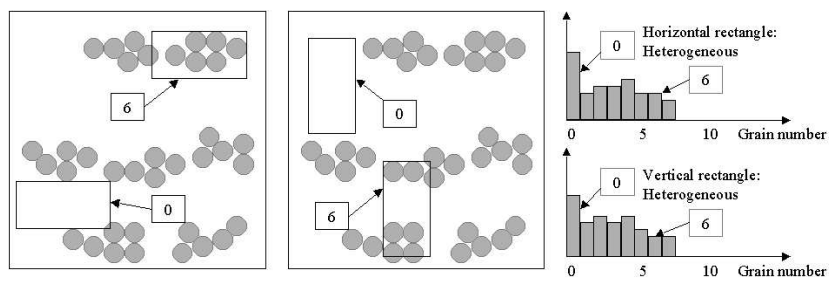

Fig. 25. Histogram of local grain number. At this scale, the structure is isotropic

In Fig. 25, histograms of local grain numbers are the same in both directions $X$ and $Y$. But, if a longer parallelepiped is considered (Fig. 26), histograms indicate anisotropy. In this last case, it is possible to find the main direction of aggregates (here the horizontal direction). With parallelepipeds showing different orientations, it is possible to study the size and orientation of aggregates.
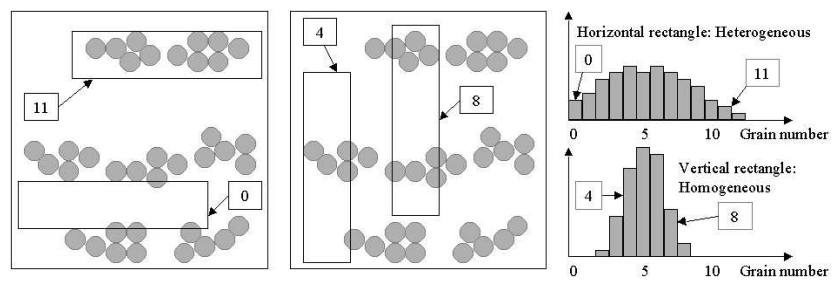

Fig. 26. Histogram of local grain number. At this scale, the structure is anisotropic

This measurement is quite fast because, for example, for an image of $1.2 \times 1.2 \times 1.2 \mathrm{~mm}^{3}$, with a resolution of $2 \mu \mathrm{m}$ and results with parallelepipeds with dimensions $64 \times 64 \times 128 \mu \mathrm{m}^{3}$ and $64 \times 64 \times$ $256 \mu \mathrm{m}^{3}$ every $32 \mu \mathrm{m}$ returns a measurement more than $10^{5}$ faster than covariance. The main advantages of this type of measurement are the description of heterogeneity and anisotropy, and the very low cost of calculation.

For illustration, Figs. 27 and 28 show measurements obtained on two different structures, materials Fred 1 and Jmc3c (Delarue and Jeulin, 2000). The first structure (Fig. 27) shows unimodal empirical distributions, as the result of the absence of aggregates; no anisotropy is detected from this figure.
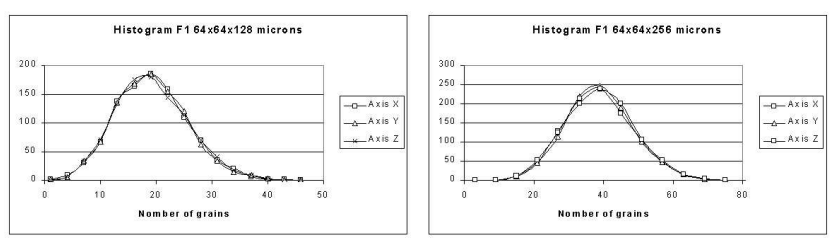

Fig. 27. Histogram of local grain number on an homogeneous and isotropic structure (Fred1)

The second structure (Fig. 28) on a material which was compressed along the $Z$ axis shows an apparent heterogeneity for the two types of histograms. The first one (left) is isotropic and corresponds to the smaller dimension of aggregates. The histogram with the larger parallelepiped (right) is sensitive to the larger dimension of the aggregates, and has a lower dispersion along the $Z$ axis. As with the covariance, this measurement can detect that aggregates are elongated orthogonally to the $Z$ axis. In a further study (Delarue and Jeulin, 2001), this kind of information is used to generate constrained simulations of spherical aggregates
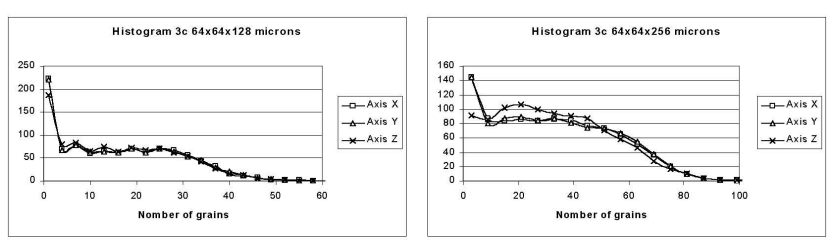

Fig. 28. Histogram of local grain number on anisotropic aggregates $(\mathrm{Jmc} 3 \mathrm{c}$ )

\section{CONCLUSION}

By means of X-ray microtomography, 3D images of materials are available. This makes it easier to study the anisotropy induced by the manufacturing process involved in the preparation of materials. Some of the characteristics studied in this paper (covariance, two point probabilities), could be measured from lower dimensional section (1D). Other morphological properties of interest introduced to study the shape and the distribution of aggregates (tortuosity, local volume fraction, geodesic distance function, orientation of pairs of inclusions, local histograms of numbers of objects) require a direct measurement on $3 \mathrm{D}$ images. 


\section{ACKNOWLEDGMENTS}

This study was sponsored by CNRS (Project 104. Programme matériaux). One of the authors (A.D.) was supported by a DGA-CNRS grant. The authors are grateful to the referees, who helped to improve earlier versions of this paper.

\section{REFERENCES}

Decker L, Jeulin D, Tovena I (1998). 3D morphological analysis of the connectivity of a porous medium. Acta Stereol 17:107-12.

Delarue A, Jeulin D (2000). Morphologie 3D de Composite Granulaires, Caractérisation Morphologique 3D de Matériaux Hétérogènes par Microtomographie $\mathrm{X}$ et Traitement d'image pour la prévision de Comportement Mécanique. Programme Matériaux du CNRS, Thème 12a, Projet $\mathrm{N}^{\circ} 104$, octobre 2000, N-29/00/MM. Paris School of Mines publication.
Delarue A (2001). Prévision du comportement électromagnétique de matériaux composites à aprtir de leur mode d'élaboration et de leur morphologie. $\mathrm{PhD}$ thesis, Ecole des Mines de Paris.

Delarue A, Jeulin D (2001). Multi-scale simulations of spherical aggregates, communication to the 8th European Congress for Stereology, Bordeaux, 4-7 September 2001. Image Anal Stereol 20: 181-6.

Matheron G (1965). Les variables régionalisées et leur estimation. Paris: Masson.

Matheron G (1975). Random sets and integral geometry. New York: J. Wiley.

Jeulin D (2001). Estimation of second and third order statistics for non-overlapping spheres. Paris School of Mines publication.

Serra J (1982). Image analysis and mathematical morphology. London: Academic Press. 